\title{
EVALUATION AND OPTIMIZATION OF ANTIOXIDANT POTENTIALITY OF XYLARIA FEEJEENSIS HMJAU22039
}

\author{
DURGA REBBAPRAGADA*, RAJAGOPAL KALYANARAMAN \\ Department of Biotechnology, School of Life Sciences, Vels University, Chennai - 600 117, Tamil Nadu, India. \\ Email: pallavi_durga@yahoo.co.in
}

Received: 27 June 2016, Revised and Accepted: 04 July 2016

\section{ABSTRACT}

Objective: Antioxidants neutralize free radicals generated in the human body and prevent them from causing damage. The present study is aimed to evaluate and optimize the antioxidant activity of Xylaria feejeensis HMJAU22039 an endophyte isolated from Tectona grandis.

Methods: The antioxidant potential was measured by 1-1diphenyl-2-picryl-hydrazil (DPPH) radical scavenging assay and nitric oxide (NO) scavenging assay. Total phenolic content (TPC) of the samples was measured by Folin-Ciocalteu reagent based assay, and the values were obtained from the regression equation: $\mathrm{y}=0.006141 \mathrm{x}+0.157733$.

Results: Potato dextrose yeast extract broth medium was selected as the basal medium as it reported a high antioxidant activity. The basal medium was optimized with $2 \%$ dextrose, $0.2 \%$ yeast extract, and $200 \mathrm{~g} / \mathrm{L}$ potato infusion. Dextrose and yeast extract were selected as carbon and nitrogen sources, respectively, as they reported high antioxidant activity. The antioxidant activity and phenolic content are highest at static condition (18.14 mg/g). Incubation temperature of $30^{\circ} \mathrm{C}$ and $\mathrm{pH}$ of the initial medium at 6 were found to be optimum conditions for high antioxidant activity. An incubation period of 20 days reported the highest antioxidant activity and phenolic content. Methanol extract recorded high antioxidant activity with a DPPH (73.86\%) followed by ethyl acetate and chloroform fractions.

Conclusion: The study highlights the importance of different physiochemical parameters in the production of secondary metabolites having antioxidant properties. The results reveal a significant positive correlation between DPPH radical scavenging assay, NO scavenging assay, and TPC.

Keywords: 1-1diphenyl-2-picryl-hydrazil, Total phenolic content, Endophytic fungus, Tectona grandis.

(C) 2016 The Authors. Published by Innovare Academic Sciences Pvt Ltd. This is an open access article under the CC BY license (http://creativecommons. org/licenses/by/4. 0/) DOI: http://dx.doi.org/10.22159/ajpcr.2016.v9s2.13734

\section{INTRODUCTION}

Natural products are naturally derived bioactive metabolites and byproducts from microorganisms, plants, or animals. These products have been exploited for human use for many years and plants have been the main source of the compounds used for medicine. Besides plants, microorganisms also constitute a major source of natural products with preferable bioactive properties. Researchers are in quest of new bioactive metabolites from new sources. Endophytic fungi appear to be one such interesting source of research. As a consequence of their contribution to the host plant, endophytes may produce a surplus of substances that may have potential use in modern medicine, agriculture, and industry. Bioactive compounds produced by endophytes have been promising prospective utility in safety and human health concerns. Endophytes provide a wide range of bioactive secondary metabolites with a unique structure, including alkaloids, benzopyranones, flavonoids, phenolic acids, quinones, terpenoids, steroids, tetralones, xanthones, and others [1]. These bioactive secondary metabolites have a wide range of application as agrochemicals, antibiotics, immunosuppressants, antiparasitics, antioxidants, and anticancer agents [2-4].

Antioxidants also called as free radical scavengers are chemicals that interact and neutralize free radicals, thus preventing them from causing damage to the organism. Free radicals are produced constantly in the human body during normal physiological processes and cause various degenerative processes such as aging, inflammation, cardiovascular diseases, atherosclerosis, diabetes, cancer, cataracts, Alzheimer's disease, and neurodegenerative disorders [5]. Antioxidants can be either used as dietary supplement or as a drug. Food industries use synthetic antioxidants such as butylated hydroxyanisole, tert-butylhydroquinone, and butylated hydroxyl toluene as food additives to prevent lipid peroxidation. Synthetic antioxidants are found to be carcinogenic and are reported to involve other toxic side effects thus compelling the search for natural antioxidants. As a result, attention has been drawn on the characterization of the antioxidant properties of products from several natural resources and isolation and identification of those important constituents. Plants and mushrooms are major sources of natural antioxidants. In recent times, fungi have emerged as the new sources of antioxidants in the form of their secondary metabolites [6]. Discovery of pestacin and isopestacin as antioxidant compounds from Pestalotiopsis microspora an endophyte in Terminalia morobensis led to the exploration of the antioxidant potential of this less explored group of fungi. Graphislactone A was isolated from Cephalosporium sp. IFB-E001, an endophytic in Trachelospermum jasminoides. The compound was confirmed to have stronger antioxidant activity in vitro as compared to butylated hydroxytoluene and ascorbic acid [7].

\section{METHODS}

\section{Isolation and identification}

Xylaria feejeensis HMJAU22039 was isolated from Tectona grandis and grown on potato dextrose agar (PDA) medium substituted with chloramphenicol. After 1 week of growth, the culture was transferred to PDA slants and stored at $4^{\circ} \mathrm{C}$. Molecular identification of the strain was confirmed by NFCCI, Pune.

\section{Antioxidant activity assays}

Free radical scavenging activity measured by 1-1diphenyl-2picryl-hydrazil

The free radical scavenging activity of all the extracts was measured by DPPH radical scavenging assay [8]. An aliquot of $1 \mathrm{ml}$ of $0.1 \mathrm{mM} \mathrm{DPPH}$ solution in methanol and $0.5 \mathrm{ml}$ of extract were mixed. The mixture was shaken vigorously and allowed to reach a steady state at room temperature for 30 minutes, and absorbance was measured at $517 \mathrm{~nm}$. The DPPH radical scavenging effect was calculated according to the 
following equation:

DPPH scavenging effect $(\%)=\frac{\mathrm{A} 0-\mathrm{A} 1}{\mathrm{~A} 0} \times 100$

Where, $\mathrm{A} 0$ is the absorbance of the control and $\mathrm{A} 1$ is the absorbance of the sample.

\section{Determination of nitric oxide scavenging activity}

An equal amount $(6 \mathrm{ml})$ of sodium nitroprusside $(5 \mathrm{mM})$ solution was mixed with $6 \mathrm{ml}$ of extract and incubated at $27^{\circ} \mathrm{C}$ for $2 \frac{1}{2} \mathrm{hrs}$. Afterward, $0.5 \mathrm{ml}$ of the reaction mixture was mixed with an equal amount of Griess reagent, and absorbance was taken at $546 \mathrm{~nm}$ [9] The nitric oxide (NO) scavenging effect was calculated according to the following equation:

NO scavenging effect $(\%)=\frac{\mathrm{A} 0-\mathrm{A} 1}{\mathrm{~A} 0} \times 100$

Where, A0 is the absorbance of the control reaction, and A1 is the absorbance of the sample.

\section{Determination of total phenolic content}

Total phenol content was estimated using Folin-Ciocalteu (FC) reagent based assay using gallic acid as standard [10]. The extract was dissolved in methanol $(1 \mathrm{mg} / \mathrm{mL})$, and $500 \mu \mathrm{L}$ of $(50 \%) \mathrm{FC}$ reagent was added followed by the addition of $1.5 \mathrm{~mL}$ of $20 \%$ of $\mathrm{Na}_{2} \mathrm{CO}_{3}$. The final volume was made up to $5 \mathrm{~mL}$ by adding distilled water. The mixture was kept at room temperature for 30 minutes, and the absorbance was recorded at $765 \mathrm{~nm}$. This procedure was also repeated to aliquots of $10-100 \mu \mathrm{g} / \mathrm{mL}$ methanolic gallic acid solutions which were used as a standard for the calibration curve. Total phenolic value of the samples was obtained from the regression equation: $\mathrm{y}=0.006141 \mathrm{x}+0.157733$ with $\mathrm{R} 2=0.9975$ and expressed as $\mathrm{mg} / \mathrm{g}$ gallic acid equivalent.

\section{Medium optimization}

\section{Standardization of basal medium}

Standardization of basal medium for optimum antioxidant activity consists of $(\mathrm{g} / \mathrm{L})$ Potato dextrose broth containing potato $(200.0 \mathrm{~g})$ and dextrose (20.0 g); Czapek-Dox Broth containing $\mathrm{NaNO}_{3}(3.0 \mathrm{~g})$, $\mathrm{K}_{2} \mathrm{HPO}_{4}(1.0 \mathrm{~g}), \mathrm{MgSO}_{4}(0.5 \mathrm{~g}), \mathrm{KCl}(0.5 \mathrm{~g}), \mathrm{FeSO}_{4}(0.01 \mathrm{~g})$, and sucrose (30.0g); Sabouraud dextrose broth containing peptone $(10 \mathrm{~g} / \mathrm{L})$ and glucose $(40 \mathrm{~g} / \mathrm{L})$; potato dextrose yeast extract broth (PDYEB) containing potato $(200.0 \mathrm{~g})$, dextrose $(20.0 \mathrm{~g})$, and yeast extract $(2.0 \mathrm{~g})$; Malt extract broth (ME) containing ME (20.0 g), peptone $(1.0 \mathrm{~g})$, and glucose $(20.0 \mathrm{~g})$ media were used. The culture was kept in static condition and an initial $\mathrm{pH}$ of 7 for all cultures. After 14 days of incubation at $26 \pm 2{ }^{\circ} \mathrm{C}$, the culture filtrate was extracted 3 times with ethyl acetate. The antioxidant activity was measured by DPPH, NO scavenging assay, and the total phenolic content (TPC) was recorded.

\section{Effect of carbon source}

Various carbon sources such as dextrose, sucrose, glucose, lactose, and maltose were amended separately into the basal medium (PDYEB) at a concentration of 2\%. X. feejeensis HMJAU22039 was inoculated to each of the media and incubated at $26 \pm 2^{\circ} \mathrm{C}$ in dark for 14 days in static condition, and their respective antioxidant activity was recorded.

\section{Effect of nitrogen source}

Various nitrogen sources such as sodium nitrate, casein, yeast extract, peptone, and ME were amended into the basal medium (PDYEB) at a concentration of nitrogen source $0.2 \%$. X. feejeensis HMJAU22039 was inoculated to the respective medium and incubated at $26 \pm 2{ }^{\circ} \mathrm{C}$ in dark for 14 days in static condition, and their respective antioxidant activity was recorded.

\section{Effect of shaking}

$X$. feejeensis was inoculated into PDYEB basal medium and was grown in static and shaking conditions. In shaking condition, the broth culture was kept on an orbital shaker at $120 \mathrm{rpm}$ and $240 \mathrm{rpm}$ for 1 week. After 14 days, the antioxidant activity and phenolic content were recorded.

\section{Effect of temperature}

The strain HMJAU22039 was inoculated into PDYEB basal medium and was grown in various ranges of temperature from 20 to $40^{\circ} \mathrm{C}$ at a difference of $5^{\circ} \mathrm{C}$ for 14 days in dark under static conditions, and the antioxidant activity and TPC were recorded.

\section{Effect of $p H$}

Initial $\mathrm{pH}$ was adjusted from 5 to 9 at a difference of one to PDYEB medium and was incubated for 14 days in dark at $26 \pm 2^{\circ} \mathrm{C}$ under static condition, and their antioxidant activity and TPC were recorded.

\section{Determination of incubation period}

The strain HMJAU22039 was inoculated into PDYEB medium and incubated up to 25 days in static condition in dark at $26 \pm 2^{\circ} \mathrm{C}$. Their antioxidant activity and TPC were measured at an interval of 5 days from the initial day of inoculation till the $25^{\text {th }}$ day.

\section{Effect of solvent}

The fungal strain was inoculated into 5 flasks containing PDYEB basal medium. The temperature was maintained at $26 \pm 2^{\circ} \mathrm{C}$ in dark. After 14 days of incubation, the culture was passed through four layers of cheesecloth to remove solids and extracted with ethyl acetate, chloroform, diethyl ether, and toluene. The fungal mat was crushed, soaked in methanol for $24 \mathrm{~h}$, and filtered. The antioxidant activity and the TPC of all the solvent extracts were recorded.

\section{RESULTS}

\section{Standardization of basal medium}

Although PDA medium was used for the isolation of $X$. feejeensis HMJAU22039, further standardization of medium showed that PDYEB was a better medium for antioxidant activity (Fig. 1). Comparative study of antioxidant activity measured by DPPH, NO scavenging activity,

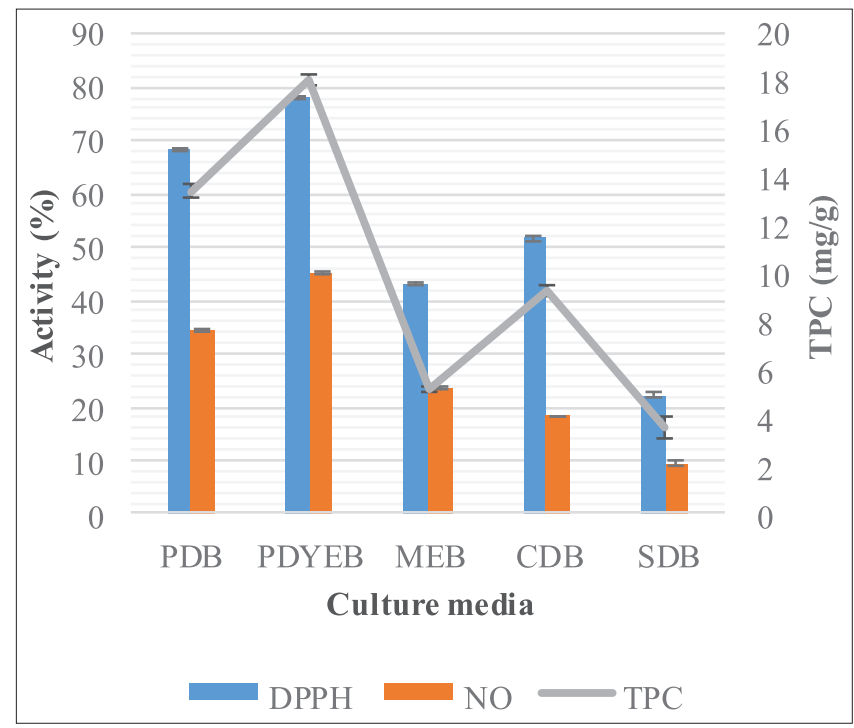

Fig. 1: Effect of different media on antioxidant potential of Xylaria feejeensis. DPPH: DPPH radical scavenging activity; NO: Nitric oxide scavenging activity; TPC: Total phenolic content. All values are reported as mean $\pm S D(n=3)$. DPPH: 1-1diphenyl-2-picrylhydrazil, NO: Nitric oxide, SD: Standard deviation 
and TPC indicated a significantly higher antioxidant activity and TPC $(18.1 \mathrm{mg} / \mathrm{g})$ in PDYEB by $X$. feejeensis compared to others. Hence, PDYEB medium was used to optimize different environmental parameters for antioxidant activity and phenol production.

\section{Effect of carbon source}

Table 1 shows the effect of carbon source on antioxidant activity and phenol content. A significantly higher value of DPPH radical scavenging (69.34\%), NO scavenging activity (38.89\%), and total phenol content $(14.31 \mathrm{mg} / \mathrm{g})$ were produced when dextrose was the carbon source. It was followed by glucose with DPPH, NO scavenging activity, and TPC at $61.38 \%, 36.41 \%$, and $11.42 \mathrm{mg} / \mathrm{g}$, respectively. Sucrose, maltose, and lactose showed moderate to low amount of antioxidant activity and phenol content.

\section{Effect of nitrogen source}

Table 2 shows the effect of nitrogen source on antioxidant activity and phenol content. A significantly higher DPPH (70.39\%), NO scavenging activity $(41.63 \%)$, and total phenol content $(16.81 \mathrm{mg} / \mathrm{g})$ were produced when yeast extract was the carbon source by $X$. feejeensis. Although sodium nitrate as nitrogen source gave good antioxidant activity, it is very less compared to that of yeast extract. ME, casein, and peptone showed very less antioxidant activity.

\section{Effect of shaking}

Table 3 shows the effect of shaking on antioxidant activity and phenolic content. A higher DPPH (71.34\%), NO scavenging activity (43.17\%), and total phenol content $(17.14 \mathrm{mg} / \mathrm{g})$ were produced when the culture was kept at static condition by X. feejeensis HMJAU22039. The antioxidant activity was lowered when the culture was kept at 120 rpm shaking condition and was significantly lowered at $240 \mathrm{rpm}$ shaking condition.

\section{Effect of temperature}

Maximum antioxidant activity of DPPH, NO scavenging, and TPC $72.41 \%, 44.14 \%$, and $14.11 \mathrm{mg} / \mathrm{g}$, respectively, was recorded at $30^{\circ} \mathrm{C}$ (Fig. 2). Low antioxidant activity and phenol content were found at very low and high temperatures $20^{\circ} \mathrm{C}$ and $40^{\circ} \mathrm{C}$, respectively. An increasing antioxidant activity was recorded at a temperature between $20^{\circ} \mathrm{C}$ and $30^{\circ} \mathrm{C}$ after which a decrease in activity and phenol content was observed.

\section{Effect of $p H$}

Medium with initial pH 6 was found to be optimal for DPPH (68.12\%), NO scavenging activity (39.03\%), and total phenol content $(16.61 \mathrm{mg} / \mathrm{g}$ ) (Fig. 3). pH 5 and pH 7 also reported good antioxidant activity. Very little antioxidant activity was observed at pH 9.

\section{Effect of incubation period}

The incubation period at 20 days was observed to be optimum for maximum antioxidant activity, with $84.32 \%, 52.14 \%$, and $24.42 \mathrm{mg} / \mathrm{g}$ for DPPH, NO scavenging, and TPC, respectively (Fig. 4). Maximum antioxidant activity was reported after the fungus reached its stationary phase after 20 days of incubation, the antioxidant activity was slightly lowered on the $25^{\text {th }}$ day of the incubation period.

\section{Effect of solvent}

Maximum antioxidant activity of DPPH, NO scavenging, and TPC at $73.86 \%, 44.97 \%$, and $18.36 \mathrm{mg} / \mathrm{g}$, respectively, by X. feejeensis was reported by methanolic extract of the culture followed by ethyl acetate and chloroform extract (Table 4). Diethyl ether and toluene reported low antioxidant activity.

\section{DISCUSSION}

Recent research showed that a number of potent antioxidants had been isolated from endophytic fungi [11-13]. Like many other metabolites
Table 1: Effect of carbon source on antioxidant potential of Xylaria feejeensis

\begin{tabular}{llll}
\hline C source & DPPH & NO scavenging & TPC \\
\hline Glucose & $61.38 \pm 0.25$ & $36.41 \pm 0.43$ & $11.42 \pm 0.35$ \\
Dextrose & $69.34 \pm 0.31$ & $38.89 \pm 0.38$ & $14.31 \pm 0.14$ \\
Maltose & $33.12 \pm 0.56$ & $18.13 \pm 0.33$ & $4.37 \pm 0.59$ \\
Sucrose & $59.64 \pm 0.12$ & $28.64 \pm 0.52$ & $8.83 \pm 0.28$ \\
Lactose & $34.39 \pm 0.18$ & $20.14 \pm 0.15$ & $2.14 \pm 0.1$ \\
\hline
\end{tabular}

All values are reported as mean \pm SD ( $n=3)$. DPPH: DPPH radical scavenging activity, NO: NO scavenging activity, TPC: Total phenolic content, SD: Standard deviation, DPPH: 1-1diphenyl-2-picryl-hydrazil, NO: Nitric oxide

Table 2: Effect of nitrogen source on antioxidant potential of Xylaria feejeensis

\begin{tabular}{llll}
\hline N source & DPPH & NO scavenging & TPC \\
\hline Yeast extract & $70.39 \pm 0.23$ & $41.63 \pm 0.27$ & $16.81 \pm 0.5$ \\
Malt extract & $34.33 \pm 0.45$ & $17.41 \pm 0.13$ & $4.73 \pm 0.16$ \\
Peptone & $38.19 \pm 0.44$ & $19.29 \pm 0.48$ & $5.97 \pm 0.22$ \\
Sodium nitrate & $49.14 \pm 0.3$ & $21.45 \pm 0.46$ & $9.36 \pm 0.39$ \\
Casein & $42.47 \pm 0.51$ & $26.39 \pm 0.57$ & $7.19 \pm 0.34$ \\
\hline
\end{tabular}

All values are reported as mean \pm SD $(n=3)$. DPPH DPPH radical scavenging activity, NO: NO scavenging activity, TPC: Total phenolic content, SD: Standard deviation, DPPH: 1-1diphenyl-2-picryl-hydrazil, NO: Nitric oxide

Table 3: Effect of shaking on antioxidant potential of Xylaria feejeensis

\begin{tabular}{llll}
\hline Condition & DPPH & NO scavenging & TPC \\
\hline Static & $71.34 \pm 0.5$ & $43.17 \pm 0.2$ & $17.14 \pm 0.18$ \\
Shaking $120 \mathrm{rpm}$ & $64.81 \pm 0.42$ & $40.23 \pm 0.37$ & $15.71 \pm 0.24$ \\
Shaking $240 \mathrm{rpm}$ & $53.91 \pm 0.45$ & $33.76 \pm 0.16$ & $11.32 \pm 0.31$ \\
\hline
\end{tabular}

All values are reported as mean $\pm \mathrm{SD}(\mathrm{n}=3)$. DPPH: DPPH radical scavenging activity, NO: NO scavenging activity, TPC: Total phenolic content, SD: Standard deviation, DPPH: 1-1diphenyl-2-picryl-hydrazil, NO: Nitric oxide



Fig. 2: Effect of incubation temperature on antioxidant potential of Xylaria feejeensis. DPPH: DPPH radical scavenging activity; NO: NO scavenging activity; TPC: Total phenolic content. All values are reported as mean \pm SD $(n=3)$. DPPH: 1-1diphenyl-2-picryl-hydrazil, NO: Nitric oxide, SD: Standard deviation

productions of antioxidant compounds such as phenols and flavonoids depend on media components and their conditions. Variation of 


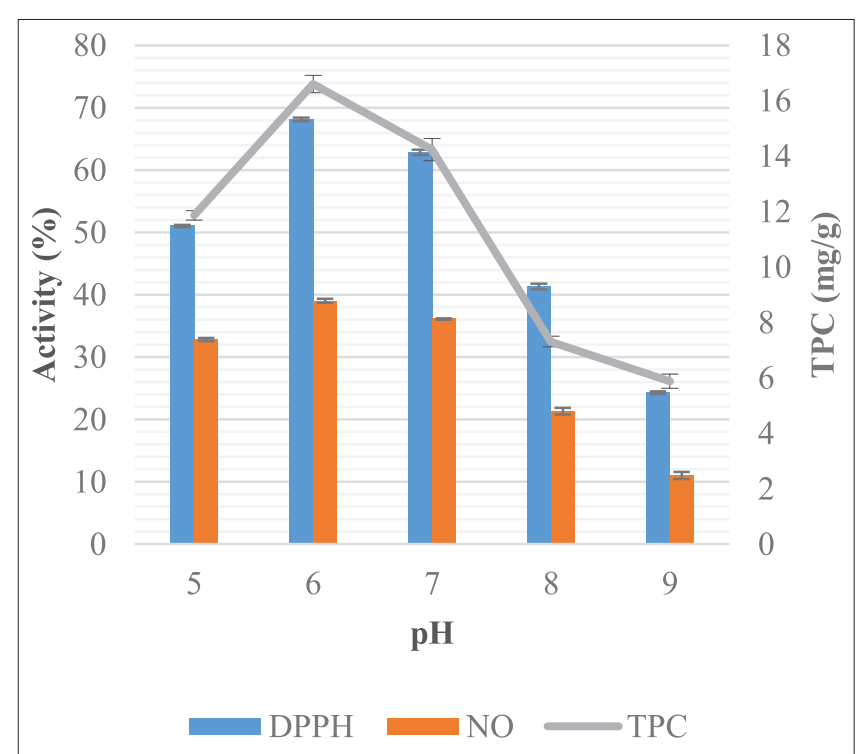

Fig. 3: Effect of pH on antioxidant potential of Xylaria feejeensis. DPPH: DPPH radical scavenging activity; NO: NO scavenging activity; TPC: Total phenolic content. All values are reported as mean $\pm S D(n=3)$. DPPH: 1-1diphenyl-2-picryl-hydrazil, NO: Nitric oxide, SD: Standard deviation

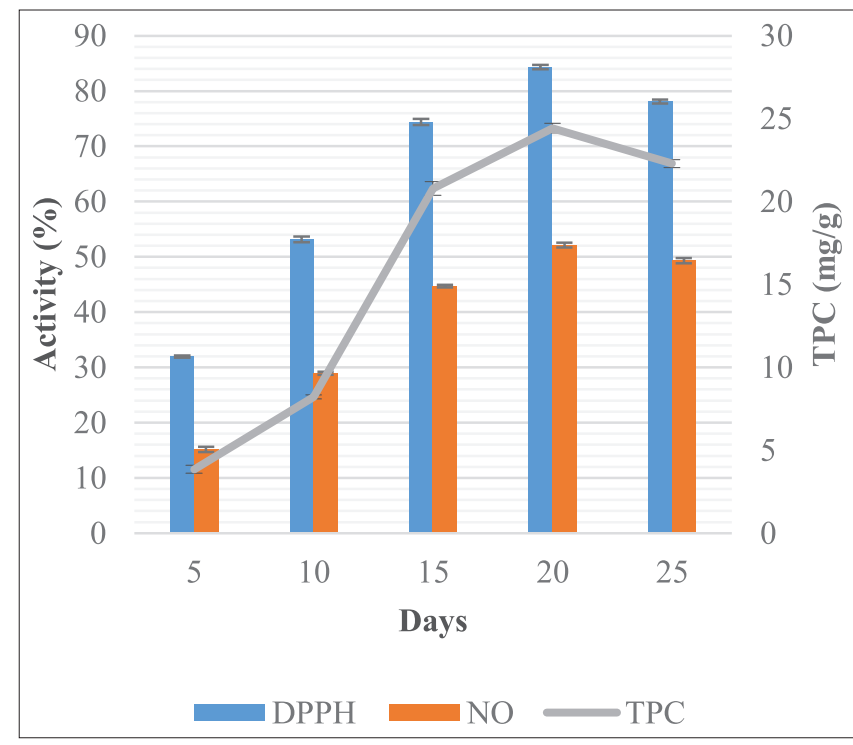

Fig. 4: Effect of incubation period on antioxidant potential of Xylaria feejeensis. DPPH: DPPH radical scavenging activity; NO: NO scavenging activity; TPC: Total phenolic content. All values are reported as mean $\pm S D(n=3)$. DPPH: 1-1diphenyl-2-picryl-hydrazil, NO: Nitric oxide, SD: Standard deviation

Table 4: Effect of extraction with different solvents on antioxidant potential of Xylaria feejeensis

\begin{tabular}{llll}
\hline Solvent & DPPH & NO scavenging & TPC \\
\hline Ethyl acetate & $69.24 \pm 0.54$ & $38.43 \pm 0.55$ & $15.02 \pm 0.32$ \\
Chloroform & $60.23 \pm 0.41$ & $31.14 \pm 0.37$ & $13.11 \pm 0.36$ \\
Diethyl ether & $39.07 \pm 0.2$ & $15.84 \pm 0.24$ & $7.89 \pm 0.11$ \\
Methanol & $73.86 \pm 0.49$ & $44.97 \pm 0.26$ & $18.36 \pm 0.4$ \\
Toluene & $21.76 \pm 0.19$ & $9.33 \pm 0.58$ & $6.29 \pm 0.17$ \\
\hline
\end{tabular}

All values are reported as mean $\pm S D(n=3)$. DPPH: DPPH radical scavenging activity, NO: NO scavenging activity, TPC: Total phenolic content, SD: Standard deviation, DPPH 1-1diphenyl-2-picryl-hydrazil, NO: Nitric oxide physiochemical and nutritional parameters such as the type of carbon and nitrogen sources, $\mathrm{pH}$, shaking, temperature, incubation period, and inoculum size of the fungal strain can greatly influence secondary metabolite biosynthesis [14]. Therefore, optimization of these parameters is a vital step for the enhancement of bioactive metabolites. The present study demonstrated the basic composition of potato dextrose yeast extract medium to be the best for the effective production of antioxidant activity of $X$. feejeensis. Dextrose and yeast extract were found to be promising carbon and nitrogen sources for obtaining good antioxidant activity by $X$. feejeensis. Many researchers found dextrose as an optimal carbon source for growth and metabolite synthesis [15]. Yeast extract as a competitive nitrogen source for optimum growth and metabolite production of another Xylaria species has been reported [16]. Potato infusion has been maintained at a constant level of $200 \mathrm{~g} / \mathrm{L}$ throughout the experimental design as components present in the infusion are required by the fungi for a variety of regulatory functions and biosynthesis of various secondary metabolites. Static culture of X. feejeensis was found to be more suitable for antioxidant activity compared to shake flask culture. This reinforces earlier observation of different researchers who have used static conditions to optimize antioxidant activity [17]. It might be caused by the low amount of phenols produced under shaking conditions, which are important for antioxidant activity. This study also demonstrated that 20 days of incubation period was optimum for antioxidant activity, and the subsequent decline in bioactivity could be due to the depletion of nutrients available for the fungi. This reduction might also be caused by the degradation of secondary metabolites (phenols) produced by fungi as demonstrated by the reduction in the TPC. Earlier studies on fast growing species such as Aspergillus, Penicillium, and Chaetomium showed 10-15 days to me the optimum incubation period for antioxidant activity [18]. The increase in the optimum incubation period for antioxidant activity of Xylaria might be due to the relatively slow growth rate of the strain. A temperature of $30^{\circ} \mathrm{C}$ was found to be optimum for antioxidant activity which is also supported by earlier studies that report temperatures between $25^{\circ} \mathrm{C}$ and $30^{\circ} \mathrm{C}$ as optimum for antioxidant activity [19]. It is assumed that low temperature ceases the metabolic activity of the fungus, and high temperature kills the cell of the fungus. Very less activity was detected at $\mathrm{pH}$ extreme and was optimally best between the $\mathrm{pH} 6$ and 7 . The present results corroborate the previous studies done on Saccharomyces cerevisiae where very less antioxidant activity was reported at $\mathrm{pH}$ extremes [20]. The $\mathrm{pH}$ of a culture is associated with permeability of the cell wall and membrane which might affects ion uptake and loss to the nutrient medium [21]. Delayed metabolite production due to delayed mycelial growth may be the reason for reduced antioxidant activity in extreme $\mathrm{pH}$ conditions.

\section{CONCLUSION}

The study highlights the importance of different media and physiochemical parameters in the production of secondary metabolites having antioxidant properties by X. feejeensis HMJAU22039. The results reveal a significant positive correlation between the DPPH radical scavenging activity, NO scavenging activity, and TPC.

\section{REFERENCES}

1. Tan RX, Zou WX. Endophytes: A rich source of functional metabolites Nat Prod Rep 2001;18(4):448-59.

2. GunatilakaAA. Natural products from plant-associated microorganisms: Distribution, structural diversity, bioactivity and implication of their occurrence. J Nat Prod 2006;69(3):509-26.

3. Ruma K, Sunil K, Prakash HS. Antioxidant, anti-inflammatory, antimicrobial and cytotoxic properties of fungal endophytes from Garcinia species. Int J Pharm Pharm Sci 2013;5(3):889-97.

4. Prabukumar S, Rajkuberan C, Ravindran K, Sivaramakrishnan S. Isolation and characterization of endophytic fungi from medicinal plant Crescentia cujete L. And their antibacterial, antioxidant and anticancer properties. Int J Pharm Pharm Sci 2015;7(11):316-21.

5. Gursoy N, Sarikurkcu C, Cengiz M, Solak MH. Antioxidant activities, metal contents, total phenolics and flavonoids of seven Morchella species. Food Chem Toxicol 2009;47(9):2381-8. 
6. Rodrigues KF, Costa GL, Carvalho MP, Epifanio RA. Evaluation of extracts produced by some tropical fungi as potential cholinesterase inhibitors. World J Microbiol Biotechnol 2005;21:1617-21.

7. Song YC, Huang WY, Sun C, Wang FW, Tan RX. Characterization of graphislactone a as the antioxidant and free radical-scavenging substance from the culture of Cephalosporium sp. IFB-E001, an endophytic fungus in Trachelospermum jasminoides. Biol Pharm Bull 2005;28(3):506-9.

8. Zhao GR, Xiang ZJ, Ye TX, Yuan YJ, Guo ZX. Antioxidant activities of Salvia miltiorrhiza and Panax notoginseng. Food Chem 2006;99:767-74.

9. Kang KS, Yokozawa T, Kim HY, Park JH. Study on the nitric oxide scavenging effects of ginseng and its compounds. J Agric Food Chem 2006;54(7):2558-62.

10. Singleton VL, Ortofehr R, Lamuela-Raventos RM. Analysis of total phenols and other oxidation substrate and antioxidants by means of Folin-Ciocalteau reagent. Methods Enzymol 1999;299:152-78.

11. Harper JK, Arif AM, Ford EJ, Strobel GA, Porco JA, Tomer DP, et al. Pestacin: A 1,3 dihydro isobenzofuran from Pestalotiopsis microspora possessing antioxidant and antimycotic activities. Tetrahedron 2003;59:2471-76

12. Strobel G, Ford E, Worapong J, Harper JK, Arif AM, Grant DM, et al. Isopestacin, an isobenzofuranone from Pestalotiopsis microspora, possessing antifungal and antioxidant activities. Phytochemistry 2002;60(2):179-83.

13. Liu X, Dong M, Chen X, Jiang M, Lv X, Yan G. Antioxidant activity and phenolics of an endophytic Xylaria sp. From Ginkgo biloba. Food Chem 2007;105(2):554-84.
14. Thakur D, Bora TC, Bordoloi GN, Maiumdar S. Influence of nutrition and culturing conditions for optimum growth and antimicrobial metabolite production by Streptomyces sp.201. J Med Mycol 2009;19:161-7.

15. Agastian P, Merlin JN, Nimal Christhudas IV, Praveen Kumar P. Optimization of growth and bioactive metabolite production: Fusarium solani. Asian J Pharm Clin Res 2013;6(3):98-103.

16. Ramesh V, Karunakaran C, Rajendran A. Optimization of submerged culture conditions for mycelial biomass production with enhanced antibacterial activity of the medicinal macro fungus Xylaria sp. Strain R006 against drug resistant bacterial pathogens. Curr Res Environ Appl Mycol 2014;4(1):88-98.

17. Arora DS, Chandra P, Kau GJ. Optimization and assay of antioxidant potential of two Penicillium spp. By different procedures. Curr Biotechnol 2012;1(1):2-10.

18. Abo-Elmagd HI. Evaluation and optimization of antioxidant potentiality of Chaetomium madrasense AUMC 9376. J Genet Eng Biotechno 2014;12(1):21-6

19. Bhattacharyya PN, Jha DK. Optimization of cultural conditions affecting growth and improved bioactive metabolite production by a subsurface Aspergillus strain TSF 146. Int J Appl Biol Pharm 2011;2(4):134-43.

20. Gazi MR, Kanda K, Kato F. Optimization of various cultural conditions on growth and antioxidant activity aeneration by Saccharomyces cerevisiae IFO 2373. J Biol Sci 2004;4(2):224-28.

21. Yamanaka T. The effect of $\mathrm{pH}$ on the growth of saprotrophic and ectomycorrhizal ammonia fungi in vitro. Mycologia 2003;95(4):584-9. 